\title{
On the Pseudorandomness of KASUMI Type Permutations *
}

\author{
Tetsu Iwata ${ }^{\dagger} \quad$ Tohru Yagi ${ }^{\ddagger} \quad$ Kaoru Kurosawa ${ }^{\dagger}$ \\ $\dagger$ Department of Computer and Information Sciences, \\ Ibaraki University \\ 4-12-1 Nakanarusawa, Hitachi, Ibaraki 316-8511, Japan \\ E-mail: $\{$ iwata, kurosawa\}@cis.ibaraki.ac.jp

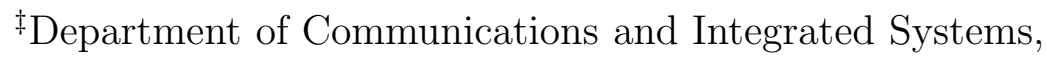 \\ Tokyo Institute of Technology \\ 2-12-1 O-okayama, Meguro, Tokyo 152-8552, Japan
}

July 3, 2003.

\begin{abstract}
KASUMI is a block cipher which has been adopted as a standard of 3GPP. In this paper, we study the pseudorandomness of idealized KASUMI type permutations for adaptive adversaries. We show that

- the four round version is pseudorandom and

- the six round version is super-pseudorandom.
\end{abstract}

Key words: Cryptography, block cipher, KASUMI, pseudorandomness, provable security.

${ }^{*}$ A preliminary version of this paper appears in The Eighth Australasian Conference on Information Security and Privacy, ACISP 2003 [5]. 


\section{Contents}

1 Introduction 1

1.1 Pseudorandomness . . . . . . . . . . . . . . . . . . . . . 1

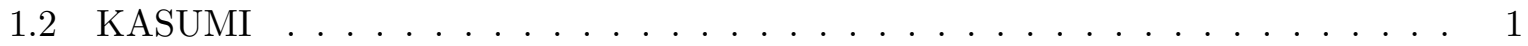

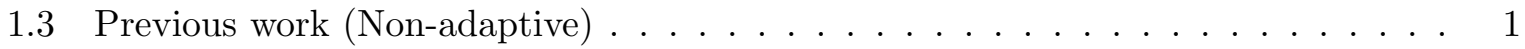

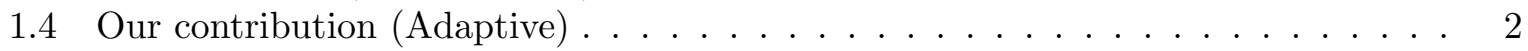

1.5 Flaw of the previous work $\ldots \ldots \ldots \ldots \ldots$

2 Preliminaries 3

2.1 Notation . . . . . . . . . . . . . . . . . . . . . . . . . 3

2.2 KASUMI type permutation $[2] \ldots \ldots \ldots \ldots \ldots \ldots$

2.3 Pseudorandom and super-pseudorandom permutations $[8] \ldots \ldots \ldots$

3 A four round KASUMI type permutation is pseudorandom 5

4 Proofs of Lemma 3.1and Lemma 3.2 7

4.1 Proof of Lemma $3.1 \ldots \ldots \ldots \ldots \ldots \ldots$

4.2 Proof of Lemma $3.2 \ldots \ldots \ldots \ldots \ldots$

5 A six round KASUMI type permutation is super-pseudorandom 14

6 Proof of Lemma 5.1 15

7 Conclusion $\quad 19$

$\begin{array}{lr}\text { References } & 20\end{array}$

A Flaws in the proof of [6] $\quad 21$

A.1 Flaws on Theorem $1 \ldots \ldots \ldots \ldots \ldots \ldots$

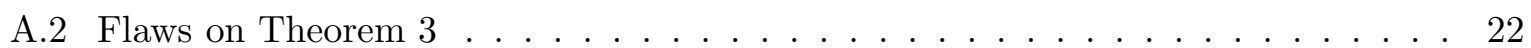




\section{Introduction}

\subsection{Pseudorandomness}

Let $R$ be a randomly chosen permutation and $\Psi$ be a block cipher such that a key is randomly chosen. We then say that

- $\Psi$ is pseudorandom if $\Psi$ and $R$ are indistinguishable and

- $\Psi$ is super-pseudorandom if $\left(\Psi, \Psi^{-1}\right)$ and $\left(R, R^{-1}\right)$ are indistinguishable.

Luby and Rackoff studied the pseudorandomness of idealized Feistel permutations, where each round function is an independent (pseudo)random function. They proved that

- the three round version is pseudorandom and

- the four round version is super-pseudorandom

for adaptive adversaries [8].

\section{$1.2 \quad$ KASUMI}

KASUMI is a block cipher which has been adopted as a standard of 3GPP [2], where 3GPP is the body standardizing the next generation of mobile telephony. The structure of KASUMI is illustrated in Fig. 1. (See [1] for details.)

- The overall structure of KASUMI is a Feistel permutation.

- Each round function consists of two functions, FL function and FO function.

- Each FO function consists of a three round MISTY type permutation, where each round function is called an FI function.

- Each FI function consists of a four round MISTY type permutation.

The initial security evaluation of KASUMI can be found in [3]. Blunden and Escott showed related key attacks on five round and six round KASUMI [4].

\subsection{Previous work (Non-adaptive)}

We idealize KASUMI as follows.

- Each FL function is ignored. (In [7], the authors stated that the security of KASUMI is mainly based on FO functions.)

- Each FI function is idealized by an independent (pseudo)random permutation.

We call such an idealized KASUMI a "KASUMI type permutation."

However, we do not assume that each FO function is a random permutation. This implies that we can not apply the result of Luby and Rackoff to KASUMI type permutations. (Indeed, Sakurai and Zheng showed that a three round MISTY type permutation is not pseudorandom [11].)

Kang et al. then showed that 

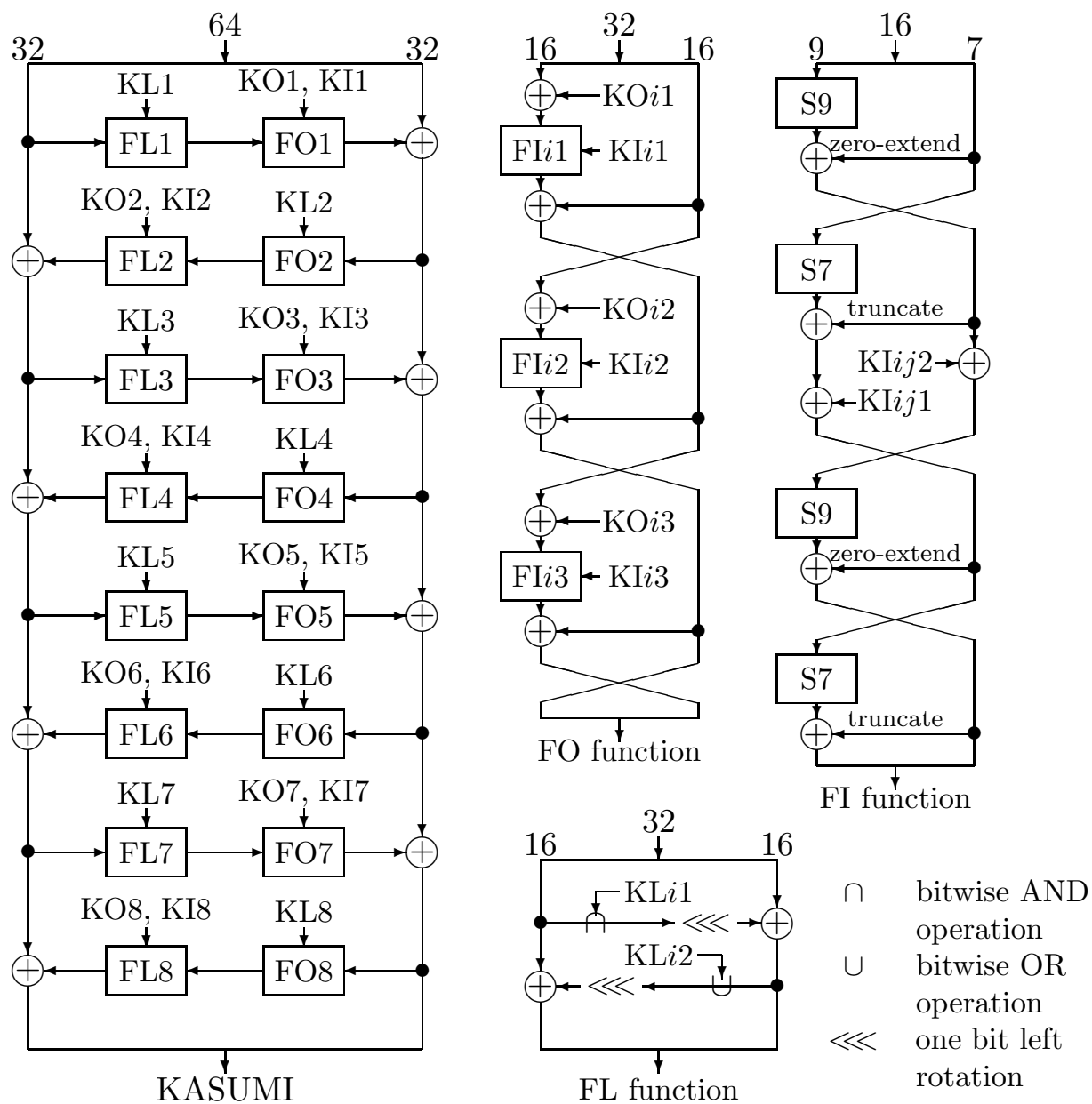

Fig. 1. KASUMI

- the three round version is not pseudorandom and

- the four round version is pseudorandom

for non-adaptive adversaries [7].

\subsection{Our contribution (Adaptive)}

In this paper, we study the pseudorandomness of KASUMI type permutations for adaptive adversaries. We prove that

- the four round version is pseudorandom and

- the six round version is super-pseudorandom.

See the following table, where $\times$ comes from [7], $\bigcirc^{1}$ comes from [7] and $\bigcirc^{2}$ is proved in this paper. 
Table 1. Summary of the previous results and our contributions.

\begin{tabular}{|l|c|c|c|c|}
\hline Number of rounds & Three & Four & Five & Six \\
\hline Pseudorandomness (non-adaptive) & $\times$ & $\bigcirc^{1}$ & $\bigcirc^{1}$ & $\bigcirc^{1}$ \\
\hline Pseudorandomness & $\times$ & $\bigcirc^{2}$ & $\bigcirc^{2}$ & $\bigcirc^{2}$ \\
\hline Super-pseudorandomness & $\times$ & $?$ & $?$ & $\bigcirc^{2}$ \\
\hline
\end{tabular}

(We cannot idealize MISTY1 [9, 10] like KASUMI type permutations because each FI function of MISTY1 is a three round MISTY type permutation and three round MISTY type permutation is not pseudorandom [11].)

\subsection{Flaw of the previous work}

Kang et al. claimed that the four round KASUMI type permutation is pseudorandom for adaptive adversaries [6]. However, we show that their proof is wrong in Appendix A.

\section{Preliminaries}

\subsection{Notation}

For a bit string $x \in\{0,1\}^{2 n}$, we denote the first (left) $n$ bits of $x$ by $x_{L}$ and the last (right) $n$ bits of $x$ by $x_{R}$. Similarly, for a bit string $x \in\{0,1\}^{4 n}$, we denote the first (left) $n$ bits of $x$ by $x_{L L}$, the next $n$ bits of $x$ by $x_{L R}$, the third $n$ bits of $x$ by $x_{R L}$, and the last (right) $n$ bits of $x$ by $x_{R R}$. That is, $x=\left(x_{L L}, x_{L R}, x_{R L}, x_{R R}\right)$. For a set of $l$-bit strings $\left\{x^{(i)} \mid x^{(i)} \in\{0,1\}^{l}\right\}_{1 \leq i \leq q}$, we say $\left\{x^{(i)}\right\}_{1 \leq i \leq q}$ are distinct to mean $x^{(i)} \neq x^{(j)}$ for $1 \leq \forall i<\forall j \leq q$.

If $S$ is a set, then $s \stackrel{R}{\leftarrow} S$ denotes the process of picking an element from $S$ uniformly at random.

Denote by $P_{n}$ the set of all permutations over $\{0,1\}^{n}$, which consists of $\left(2^{n}\right)$ ! permutations in total. For functions $f$ and $g, g \circ f$ denotes the function $x \mapsto g(f(x))$.

\section{$2.2 \quad$ KASUMI type permutation [2]}

We define KASUMI type permutations as follows.

Definition 2.1 (The basic KASUMI type permutation) Let $x \in\{0,1\}^{4 n}$. For any permutations $p_{1}, p_{2}, p_{3} \in P_{n}$, define the basic KASUMI type permutation $\psi_{p_{1}, p_{2}, p_{3}} \in P_{4 n}$ as

$$
\psi_{p_{1}, p_{2}, p_{3}}(x) \stackrel{\text { def }}{=} y
$$

where

$$
\left\{\begin{array}{l}
y_{L L} \stackrel{\text { def }}{=} x_{R L}, \\
y_{L R} \stackrel{\text { def }}{=} x_{R R}, \\
y_{R L} \stackrel{\text { def }}{=} x_{R L} \oplus p_{1}\left(x_{R R}\right) \oplus p_{2}\left(x_{R L}\right) \oplus p_{3}\left(x_{R L} \oplus p_{1}\left(x_{R R}\right)\right) \oplus x_{L L}, \text { and } \\
y_{R R} \stackrel{\text { def }}{=} x_{R L} \oplus p_{1}\left(x_{R R}\right) \oplus p_{2}\left(x_{R L}\right) \oplus x_{L R} .
\end{array}\right.
$$




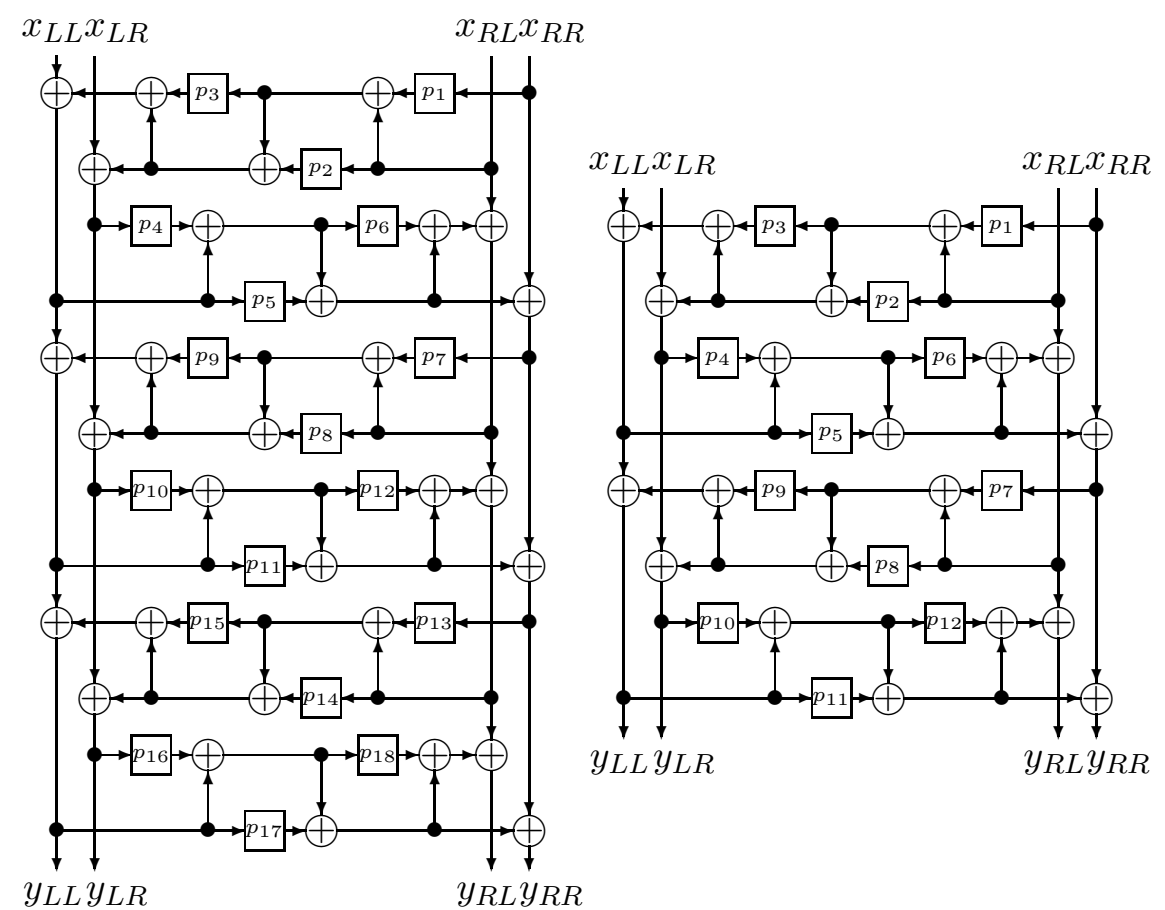

Fig. 2. A six round KASUMI type permutation $\psi\left(p_{1}, \ldots, p_{18}\right)$ (left) and a four round KASUMI type permutation $\psi\left(p_{1}, \ldots, p_{15}\right)$ (right).

Note that it is a permutation since $\psi_{p_{1}, p_{2}, p_{3}}^{-1}(y)=x$, where

$$
\left\{\begin{array}{l}
x_{L L}=y_{L L} \oplus p_{1}\left(y_{L R}\right) \oplus p_{2}\left(y_{L L}\right) \oplus p_{3}\left(y_{L L} \oplus p_{1}\left(y_{L R}\right)\right) \oplus y_{R L} \\
x_{L R}=y_{L L} \oplus p_{1}\left(y_{L R}\right) \oplus p_{2}\left(y_{L L}\right) \oplus y_{R R} \\
x_{R L}=y_{L L}, \text { and } \\
x_{R R}=y_{L R} .
\end{array}\right.
$$

Definition 2.2 (The $\boldsymbol{r}$ round KASUMI type permutation) Let $r \geq 1$ be an integer, and $p_{1}, p_{2}, \ldots, p_{3 r} \in P_{n}$ be permutations.

Define the $r$ round KASUMI type permutation $\psi\left(p_{1}, p_{2}, \ldots, p_{3 r}\right) \in P_{4 n}$ as

$$
\psi\left(p_{1}, p_{2}, \ldots, p_{3 r}\right) \stackrel{\text { def }}{=} \psi_{p_{3 r-2}, p_{3 r-1}, p_{3 r}} \circ \psi_{p_{3 r-5}, p_{3 r-4}, p_{3 r-3}} \circ \cdots \circ \psi_{p_{1}, p_{2}, p_{3}} .
$$

See Fig. 2 for illustrations. For simplicity, swaps are omitted.

\subsection{Pseudorandom and super-pseudorandom permutations [8]}

Our adaptive adversary $\mathcal{A}$ is modeled as a Turing machine that has black-box access to an oracle (or oracles). The computational power of $\mathcal{A}$ is unlimited, but the total number of oracle calls is limited to a parameter $q$. After making at most $q$ queries to the oracle(s) adaptively, $\mathcal{A}$ outputs a bit.

The pseudorandomness of a block cipher $\Psi$ over $\{0,1\}^{4 n}$ captures its computational indistinguishability from $P_{4 n}$, where the adversary is given access to the forward direction of the 
permutation. In other words, it measures security of a block cipher against adaptive chosen plaintext attack.

Definition 2.3 (Advantage, prp) Let a block cipher $\Psi$ be a family of permutations over $\{0,1\}^{4 n}$. Let $\mathcal{A}$ be an adversary. Then $\mathcal{A}$ 's advantage is defined by

$$
\operatorname{Adv}_{\Psi}^{\text {prp }}(\mathcal{A}) \stackrel{\text { def }}{=}\left|\operatorname{Pr}\left(\psi \stackrel{R}{\leftarrow} \Psi: \mathcal{A}^{\psi}=1\right)-\operatorname{Pr}\left(R \stackrel{R}{\leftarrow} P_{4 n}: \mathcal{A}^{R}=1\right)\right| .
$$

The notation $\mathcal{A}^{\psi}$ indicates $\mathcal{A}$ with an oracle which, in response to a query $x$, returns $y \leftarrow \psi(x)$. The notation $\mathcal{A}^{R}$ indicates $\mathcal{A}$ with an oracle which, in response to a query $x$, returns $y \leftarrow R(x)$.

The super-pseudorandomness of a block cipher $\Psi$ over $\{0,1\}^{4 n}$ captures its computational indistinguishability from $P_{4 n}$, where the adversary is given access to both directions of the permutation. In other words, it measures security of a block cipher against adaptive chosen plaintext and chosen ciphertext attacks.

Definition 2.4 (Advantage, sprp) Let a block cipher $\Psi$ be a family of permutations over $\{0,1\}^{4 n}$. Let $\mathcal{A}$ be an adversary. Then $\mathcal{A}$ 's advantage is defined by

$$
\operatorname{Adv}_{\Psi}^{\text {sprp }}(\mathcal{A}) \stackrel{\text { def }}{=}\left|\operatorname{Pr}\left(\psi \stackrel{R}{\leftarrow} \Psi: \mathcal{A}^{\psi, \psi^{-1}}=1\right)-\operatorname{Pr}\left(R \stackrel{R}{\longleftarrow} P_{4 n}: \mathcal{A}^{R, R^{-1}}=1\right)\right| .
$$

The notation $\mathcal{A}^{\psi, \psi^{-1}}$ indicates $\mathcal{A}$ with an oracle which, in response to a query $(+, x)$, returns $y \leftarrow \psi(x)$, and in response to a query $(-, y)$, returns $x \leftarrow \psi^{-1}(y)$. The notation $\mathcal{A}^{R, R^{-1}}$ indicates $\mathcal{A}$ with an oracle which, in response to a query $(+, x)$, returns $y \leftarrow R(x)$, and in response to a query $(-, y)$, returns $x \leftarrow R^{-1}(y)$.

\section{A four round KASUMI type permutation is pseudorandom}

Theorem 3.1 For $1 \leq i \leq 12$, let $p_{i} \in P_{n}$ be a random permutation. Let $\psi=\psi\left(p_{1}, \ldots, p_{12}\right)$ be a four round KASUMI type permutation, $R \in P_{4 n}$ be a random permutation, and $\Psi \stackrel{\text { def }}{=}\{\psi \mid$ $\psi=\psi\left(p_{1}, \ldots, p_{12}\right), p_{i} \in P_{n}$ for $\left.1 \leq i \leq 12\right\}$.

Then for any adversary $\mathcal{A}$ that makes at most q queries in total,

$$
\operatorname{Adv}_{\Psi}^{\mathrm{prp}}(\mathcal{A}) \leq \frac{15}{2} \cdot \frac{q(q-1)}{2^{n}-1}
$$

Proof. Let $\mathcal{O}$ be either $R$ or $\psi$. The adversary $\mathcal{A}$ has oracle access to $\mathcal{O}$. $\mathcal{A}$ can make a query $x$ and the oracle returns $y=\mathcal{O}(x)$. For the $i$-th query $\mathcal{A}$ makes to $\mathcal{O}$, define the query-answer pair $\left(x^{(i)}, y^{(i)}\right) \in\{0,1\}^{4 n} \times\{0,1\}^{4 n}$, where $\mathcal{A}$ 's query was $x^{(i)}$ and the answer it got was $y^{(i)}$. Define view $v$ of $\mathcal{A}$ as $v=\left\langle\left(x^{(1)}, y^{(1)}\right), \ldots,\left(x^{(q)}, y^{(q)}\right)\right\rangle$. We say that $v=\left\langle\left(x^{(1)}, y^{(1)}\right), \ldots,\left(x^{(q)}, y^{(q)}\right)\right\rangle$ is a possible view if there exists some permutation $p \in P_{4 n}$ such that $p\left(x^{(i)}\right)=y^{(i)}$ for $1 \leq \forall i \leq q$ (or, equivalently, $v=\left\langle\left(x^{(1)}, y^{(1)}\right), \ldots,\left(x^{(q)}, y^{(q)}\right)\right\rangle$ is a possible view if $\left\{x^{(i)}\right\}_{1 \leq i \leq q}$ are distinct and $\left\{y^{(i)}\right\}_{1 \leq i \leq q}$ are distinct).

Since $\mathcal{A}$ is computationally unbounded, we may without loss of generality assume that $\mathcal{A}$ is deterministic. This implies that for every $1 \leq i \leq q$ the $i$-th query $x^{(i)}$ is fully determined 
by the first $i-1$ query-answer pairs, and the final output of $\mathcal{A}(0$ or 1$)$ depends only on $v$. Therefore, there exists a function $\mathcal{C}_{\mathcal{A}}(\cdot)$ such that

$$
\left\{\begin{array}{l}
\mathcal{C}_{\mathcal{A}}\left(x^{(1)}, y^{(1)}, \ldots, x^{(i-1)}, y^{(i-1)}\right)=x^{(i)} \text { for } 1 \leq i \leq q \text { and } \\
\mathcal{C}_{\mathcal{A}}(v)=\mathcal{A} \text { 's final output. }
\end{array}\right.
$$

Let $\boldsymbol{v}_{\text {one }} \stackrel{\text { def }}{=}\left\{v \mid \mathcal{C}_{\mathcal{A}}(v)=1\right\}$ and $N_{\text {one }} \stackrel{\text { def }}{=} \# \boldsymbol{v}_{\text {one }}$. Further, we let $\boldsymbol{v}_{\text {good }}$ be a set of all possible view $v=\left\langle\left(x^{(1)}, y^{(1)}\right), \ldots,\left(x^{(q)}, y^{(q)}\right)\right\rangle$ which satisfies the following four conditions:

- $\mathcal{C}_{\mathcal{A}}(v)=1$,

- $\left\{y_{L L}^{(i)}\right\}_{1 \leq i \leq q}$ are distinct,

- $\left\{y_{L R}^{(i)}\right\}_{1 \leq i \leq q}$ are distinct, and

- $\left\{x_{L L}^{(i)} \oplus x_{L R}^{(i)} \oplus y_{L L}^{(i)} \oplus y_{L R}^{(i)}\right\}_{1 \leq i \leq q}$ are distinct.

We also let $N_{\text {good }} \stackrel{\text { def }}{=} \# \boldsymbol{v}_{\text {good }}$.

Evaluation of $p_{R}$. We first evaluate $p_{R} \stackrel{\text { def }}{=} \operatorname{Pr}\left(R \stackrel{R}{\leftarrow} P_{4 n}: \mathcal{A}^{R}=1\right)$. We have $p_{R}=$ $\frac{\#\left\{R \mid \mathcal{A}^{R}=1\right\}}{\left(2^{4 n}\right) !}$. For each $v \in \boldsymbol{v}_{\text {one }}$, the number of $R$ such that

$$
R\left(x^{(i)}\right)=y^{(i)} \text { for } 1 \leq \forall i \leq q
$$

is exactly $\left(2^{4 n}-q\right)$ !. Therefore, we have $p_{R}=\sum_{v \in \boldsymbol{v}_{\text {one }}} \frac{\#\{R \mid R \text { satisfying }(1)\}}{\left(2^{4 n}\right) !}=N_{\text {one }} \cdot \frac{\left(2^{4 n}-q\right) !}{\left(2^{4 n}\right) !}$.

Evaluation of $p_{\psi}$. We evaluate $p_{\psi} \stackrel{\text { def }}{=} \operatorname{Pr}\left(\psi \stackrel{R}{\leftarrow} \Psi: \mathcal{A}^{\psi, \psi^{-1}}=1\right)$. Note that " $\psi \stackrel{R}{\leftarrow} \Psi$ " is equivalent to " $p_{i} \stackrel{R}{\leftarrow} P_{n}$ for $1 \leq i \leq 12$ and then let $\psi \leftarrow \psi\left(p_{1}, \ldots, p_{12}\right)$." We have $p_{\psi}=$ $\frac{\#\left\{\left(p_{1}, \ldots, p_{12}\right) \mid \mathcal{A}^{\psi, \psi^{-1}}=1\right\}}{\left\{\left(2^{n}\right) !\right\}^{12}}$.

We have the following lemma. A proof of this lemma is given in Section 4.1.

Lemma 3.1 (Main Lemma for $\psi\left(p_{1}, \ldots, p_{12}\right)$ ) For any fixed possible view

$$
v=\left\langle\left(x^{(1)}, y^{(1)}\right), \ldots,\left(x^{(q)}, y^{(q)}\right)\right\rangle
$$

such that $\left\{y_{L L}^{(i)}\right\}_{1 \leq i \leq q}$ are distinct, $\left\{y_{L R}^{(i)}\right\}_{1 \leq i \leq q}$ are distinct, and $\left\{x_{L L}^{(i)} \oplus x_{L R}^{(i)} \oplus y_{L L}^{(i)} \oplus y_{L R}^{(i)}\right\}_{1 \leq i \leq q}$ are distinct, the number of $\left(p_{1}, \ldots, p_{12}\right)$ which satisfies

$$
\psi\left(x^{(i)}\right)=y^{(i)} \text { for } 1 \leq \forall i \leq q
$$

is at least $\left(1-\frac{6 q(q-1)}{2^{n}-1}\right) \cdot\left\{\left(2^{n}\right) !\right\}^{8} \cdot\left\{\left(2^{n}-q\right) !\right\}^{4}$.

Then from Lemma 3.1, we have

$$
\begin{aligned}
p_{\psi} & \geq \sum_{v \in \boldsymbol{v}_{\text {good }}} \frac{\#\left\{\left(p_{1}, \ldots, p_{12}\right) \mid\left(p_{1}, \ldots, p_{12}\right) \text { satisfying }(2)\right\}}{\left\{\left(2^{n}\right) !\right\}^{12}} \\
& \geq \sum_{v \in \boldsymbol{v}_{\text {good }}}\left(1-\frac{6 q(q-1)}{2^{n}-1}\right) \cdot \frac{\left\{\left(2^{n}-q\right) !\right\}^{4}}{\left\{\left(2^{n}\right) !\right\}^{4}} .
\end{aligned}
$$

Now we have the following lemma. See Section 4.2 for a proof. 
Lemma $3.2 N_{\text {good }} \geq N_{\text {one }}-\frac{3}{2} \cdot \frac{q(q-1)}{2^{n}-1} \cdot \frac{\left(2^{4 n}\right) !}{\left(2^{4 n}-q\right) !}$.

From Lemma 3.2, we have

$$
\begin{aligned}
p_{\psi} & \geq\left(N_{\text {one }}-\frac{3}{2} \cdot \frac{q(q-1)}{2^{n}-1} \cdot \frac{\left(2^{4 n}\right) !}{\left(2^{4 n}-q\right) !}\right) \cdot\left(1-\frac{6 q(q-1)}{2^{n}-1}\right) \cdot \frac{\left\{\left(2^{n}-q\right) !\right\}^{4}}{\left\{\left(2^{n}\right) !\right\}^{4}} \\
& =\left(p_{R}-\frac{3}{2} \cdot \frac{q(q-1)}{2^{n}-1}\right) \cdot\left(1-\frac{6 q(q-1)}{2^{n}-1}\right) \cdot \frac{\left\{\left(2^{n}-q\right) !\right\}^{4}}{\left\{\left(2^{n}\right) !\right\}^{4}} \cdot \frac{\left\{\left(2^{4 n}\right) !\right\}}{\left\{\left(2^{4 n}-q\right) !\right\}} .
\end{aligned}
$$

Now it is easy to see that $\frac{\left\{\left(2^{n}-q\right) !\right\}^{4}}{\left\{\left(2^{n}\right) !\right\}^{4}} \cdot \frac{\left\{\left(2^{4 n}\right) !\right\}}{\left\{\left(2^{4 n}-q\right) !\right\}} \geq 1$ (this can be shown easily by an induction on $q)$. Then $p_{\psi} \geq\left(p_{R}-\frac{3}{2} \cdot \frac{q(q-1)}{2^{n}-1}\right) \cdot\left(1-\frac{6 q(q-1)}{2^{n}-1}\right) \geq p_{R}-\frac{15}{2} \cdot \frac{q(q-1)}{2^{n}-1}$. Applying the same argument to $1-p_{\psi}$ and $1-p_{R}$ yields that $1-p_{\psi} \geq 1-p_{R}-\frac{15}{2} \cdot \frac{q(q-1)}{2^{n}-1}$, and we have $\left|p_{\psi}-p_{R}\right| \leq \frac{15}{2} \cdot \frac{q(q-1)}{2^{n}-1}$.

From Theorem 3.1, it is straightforward to show that $\psi=\psi\left(p_{1}, \ldots, p_{12}\right)$ is pseudorandom even if each $p_{i}$ is a pseudorandom permutation by using a standard hybrid argument. For example, see [8].

\section{Proofs of Lemma 3.1 and Lemma 3.2}

\subsection{Proof of Lemma 3.1}

First, we need the following lemma.

Lemma 4.1 For $1 \leq i \leq q$, let $X^{(i)}=\left(X_{L}^{(i)}, X_{R}^{(i)}\right) \in\{0,1\}^{2 n}$ be fixed bit strings such that $\left\{X_{L}^{(i)}\right\}_{1 \leq i \leq q}$ are distinct and $\left\{X_{R}^{(i)}\right\}_{1 \leq i \leq q}$ are distinct. Similarly, for $1 \leq i \leq q$, let $Y^{(i)}=\left(Y_{L}^{(i)}, Y_{R}^{(i)}\right) \in\{0,1\}^{2 n}$ be fixed bit strings such that $\left\{Y_{L}^{(i)} \oplus Y_{R}^{(i)}\right\}_{1 \leq i \leq q}$ are distinct. Let $P_{1}, P_{2}, P_{3} \in P_{n}$ be permutations. Then the number of $\left(P_{1}, P_{2}, P_{3}\right)$ such that

- $P_{1}\left(X_{L}^{(i)}\right) \oplus X_{R}^{(i)} \oplus P_{2}\left(X_{R}^{(i)}\right)=Y_{R}^{(i)}$ for $1 \leq \forall i \leq q$, and

- $P_{3}\left(P_{1}\left(X_{L}^{(i)}\right) \oplus X_{R}^{(i)}\right) \oplus P_{1}\left(X_{L}^{(i)}\right) \oplus X_{R}^{(i)} \oplus P_{2}\left(X_{R}^{(i)}\right)=Y_{L}^{(i)}$ for $1 \leq \forall i \leq q$

is at least $\left(1-\frac{q(q-1)}{2^{n}-1}\right) \cdot\left(2^{n}\right) ! \cdot\left\{\left(2^{n}-q\right) !\right\}^{2}$.

See Fig. 3 for an illustration.

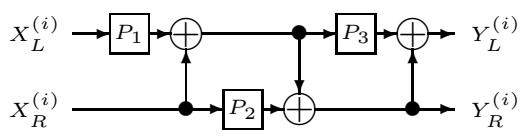

Fig. 3. Illustration of the conditions in Lemma 4.1. 
Proof. First observe that the number of $P_{1}$ such that

$$
P_{1}\left(X_{L}^{(i)}\right) \oplus X_{R}^{(i)} \oplus Y_{R}^{(i)}=P_{1}\left(X_{L}^{(j)}\right) \oplus X_{R}^{(j)} \oplus Y_{R}^{(j)} \text { for } 1 \leq \exists i<\exists j \leq q
$$

is at most $\left(\begin{array}{l}q \\ 2\end{array}\right) \cdot \frac{\left\{\left(2^{n}\right) !\right\}}{2^{n}-1}$, since $X_{L}^{(i)} \neq X_{L}^{(j)}$ for $1 \leq \forall i<\forall j \leq q$.

Next we see that the number of $P_{1}$ such that

$$
P_{1}\left(X_{L}^{(i)}\right) \oplus X_{R}^{(i)}=P_{1}\left(X_{L}^{(j)}\right) \oplus X_{R}^{(j)} \text { for } 1 \leq \exists i<\exists j \leq q
$$

is at most $\left(\begin{array}{l}q \\ 2\end{array}\right) \cdot \frac{\left\{\left(2^{n}\right) !\right\}}{2^{n}-1}$, since $X_{L}^{(i)} \neq X_{L}^{(j)}$ for $1 \leq \forall i<\forall j \leq q$.

We now fix any $P_{1}$ which does not satisfy either (3) or (4). We have at least $\left(2^{n}\right) ! \cdot\left(1-\frac{q(q-1)}{2^{n}-1}\right)$ choice of such $P_{1}$. This implies that $P_{1}$ is fixed in such a way that $\left\{P_{1}\left(X_{L}^{(i)}\right) \oplus X_{R}^{(i)} \oplus Y_{R}^{(i)}\right\}_{1 \leq i \leq q}$ (which are the outputs of $P_{2}$ ) are distinct, and $\left\{P_{1}\left(X_{L}^{(i)}\right) \oplus X_{R}^{(i)}\right\}_{1 \leq i \leq q}$ (which are the inputs to $\left.P_{3}\right)$ are distinct.

We know from our condition that $\left\{X_{R}^{(i)}\right\}_{1 \leq i \leq q}$ (which are the inputs of $P_{2}$ ) are distinct, and $\left\{Y_{L}^{(i)} \oplus Y_{R}^{(i)}\right\}_{1 \leq i \leq q}$ (which are the outputs of $P_{3}$ ) are distinct. Therefore, we have exactly $\left(2^{n}-q\right)$ ! choice of $P_{2}$ and $\left(2^{n}-q\right)$ ! choice of $P_{3}$ for any such fixed $P_{1}$.

Q.E.D.

Now for $1 \leq i \leq q$ and $1 \leq j \leq 12$, let $I_{j}^{(i)}$ denote the input to $p_{i}$ when the input to $\phi$ is $x^{(i)}$ and the output is $y^{(i)}$. Similarly, let $O_{j}^{(i)}$ denote the output of $p_{i}$ when the input to $\phi$ is $x^{(i)}$ and the output is $y^{(i)}$.

We next have the following lemma.

Lemma 4.2 For any fixed possible view $v=\left\langle\left(x^{(1)}, y^{(1)}\right), \ldots,\left(x^{(q)}, y^{(q)}\right)\right\rangle$, the number of $\left(p_{1}, p_{2}, p_{3}, p_{4}\right)$ such that

$$
I_{6}^{(i)}=I_{6}^{(j)} \text { or } I_{6}^{(i)} \oplus x_{R R}^{(i)}=I_{6}^{(j)} \oplus x_{R R}^{(j)}, \text { for } 1 \leq \exists i<\exists j \leq q
$$

is at most $\frac{2 q(q-1)}{2^{n}-1} \cdot\left\{\left(2^{n}\right) !\right\}^{4}$.

Proof. First, we fix $i$ and $j$ such that $1 \leq i<j \leq q$, and consider the condition

$$
I_{6}^{(i)}=I_{6}^{(j)} \text { or } I_{6}^{(i)} \oplus x_{R R}^{(i)}=I_{6}^{(j)} \oplus x_{R R}^{(j)}
$$

in the following four cases:

Case $x_{R R}^{(i)} \neq x_{R R}^{(j)}$. First, consider the condition

$$
p_{1}\left(x_{R R}^{(i)}\right) \oplus x_{R L}^{(i)} \oplus x_{L R}^{(i)}=p_{1}\left(x_{R R}^{(j)}\right) \oplus x_{R L}^{(j)} \oplus x_{L R}^{(j)} .
$$

The number of $p_{1}$ which satisfies (7) is at most $\frac{\left(2^{n}\right) !}{2^{n}-1}$ since $x_{R R}^{(i)} \neq x_{R R}^{(j)}$, and thus we have

$$
\#\left\{\left(p_{1}, \ldots, p_{4}\right) \mid\left(p_{1}, \ldots, p_{4}\right) \text { satisfies both }(6) \text { and }(7)\right\} \leq \frac{\left\{\left(2^{n}\right) !\right\}^{4}}{2^{n}-1} .
$$

Next, consider any $p_{1}$ which does not satisfy (7), that is,

$$
p_{1}\left(x_{R R}^{(i)}\right) \oplus x_{R L}^{(i)} \oplus x_{L R}^{(i)} \neq p_{1}\left(x_{R R}^{(j)}\right) \oplus x_{R L}^{(j)} \oplus x_{L R}^{(j)} .
$$


For this $p_{1}$, we consider the condition

$$
p_{2}\left(x_{R L}^{(i)}\right) \oplus p_{1}\left(x_{R R}^{(i)}\right) \oplus x_{R L}^{(i)} \oplus x_{L R}^{(i)}=p_{2}\left(x_{R L}^{(j)}\right) \oplus p_{1}\left(x_{R R}^{(j)}\right) \oplus x_{R L}^{(j)} \oplus x_{L R}^{(j)},
$$

which is equivalent to $I_{4}^{(i)}=I_{4}^{(j)}$. Since (9) holds, the number of $p_{2}$ which satisfies (10) is at most $\frac{\left(2^{n}\right) !}{2^{n}-1}$, and thus we have

$$
\#\left\{\left(p_{1}, \ldots, p_{4}\right) \mid\left(p_{1}, \ldots, p_{4}\right) \text { satisfies }(6),(9) \text { and }(10)\right\} \leq \frac{\left\{\left(2^{n}\right) !\right\}^{4}}{2^{n}-1}
$$

Next, consider any $p_{1}$ which satisfies (9), and any $p_{2}$ which does not satisfy (10). That is,

$$
p_{2}\left(x_{R L}^{(i)}\right) \oplus p_{1}\left(x_{R R}^{(i)}\right) \oplus x_{R L}^{(i)} \oplus x_{L R}^{(i)} \neq p_{2}\left(x_{R L}^{(j)}\right) \oplus p_{1}\left(x_{R R}^{(j)}\right) \oplus x_{R L}^{(j)} \oplus x_{L R}^{(j)}
$$

which is equivalent to $I_{4}^{(i)} \neq I_{4}^{(j)}$. For these $p_{1}, p_{2}$ and any $p_{3}$, the number of $p_{4}$ which satisfies

$$
p_{4}\left(I_{4}^{(i)}\right) \oplus I_{5}^{(i)}=p_{4}\left(I_{4}^{(j)}\right) \oplus I_{5}^{(i)},
$$

which is equivalent to $I_{6}^{(i)}=I_{6}^{(j)}$, is at most $\frac{\left(2^{n}\right) !}{2^{n}-1}$, and the number of $p_{4}$ which satisfies

$$
p_{4}\left(I_{4}^{(i)}\right) \oplus I_{5}^{(i)} \oplus x_{R R}^{(i)}=p_{4}\left(I_{4}^{(j)}\right) \oplus I_{5}^{(i)} \oplus x_{R R}^{(j)},
$$

which is equivalent to $I_{6}^{(i)} \oplus x_{R R}^{(i)}=I_{6}^{(j)} \oplus x_{R R}^{(j)}$, is at most $\frac{\left(2^{n}\right) !}{2^{n}-1}$. Therefore

$$
\#\left\{\left(p_{1}, \ldots, p_{4}\right) \mid\left(p_{1}, \ldots, p_{4}\right) \text { satisfies }(6),(9) \text { and }(12)\right\} \leq \frac{2 \cdot\left\{\left(2^{n}\right) !\right\}^{4}}{2^{n}-1} \text {. }
$$

Thus, from (8), (11) and (15), we have

$$
\#\left\{\left(p_{1}, \ldots, p_{4}\right) \mid\left(p_{1}, \ldots, p_{4}\right) \text { satisfies }(6)\right\} \leq \frac{4 \cdot\left\{\left(2^{n}\right) !\right\}^{4}}{2^{n}-1} .
$$

Case $x_{R L}^{(i)} \neq x_{R L}^{(j)}$ and $x_{R R}^{(i)}=x_{R R}^{(j)}$. For any $p_{1}$, the number of $p_{2}$ which satisfies (10) is at most $\frac{\left(2^{n}\right) !}{2^{n}-1}$ since $x_{R L}^{(i)} \neq x_{R L}^{(j)}$, and thus we have

$$
\#\left\{\left(p_{1}, \ldots, p_{4}\right) \mid\left(p_{1}, \ldots, p_{4}\right) \text { satisfies }(6) \text { and }(10)\right\} \leq \frac{\left\{\left(2^{n}\right) !\right\}^{4}}{2^{n}-1} .
$$

Next, for any $p_{1}$, any $p_{2}$ which satisfies (12), and any $p_{3}$, the number of $p_{4}$ which satisfies (13) is at most $\frac{\left(2^{n}\right) !}{2^{n}-1}$. Note that (13) is equivalent to (14) in this case. Therefore we have

$$
\#\left\{\left(p_{1}, \ldots, p_{4}\right) \mid\left(p_{1}, \ldots, p_{4}\right) \text { satisfies }(6) \text { and }(12)\right\} \leq \frac{\left\{\left(2^{n}\right)^{2^{n}}\right\}^{4}}{2^{n}} \text {. }
$$

Thus, from (17) and (18), we have

$$
\#\left\{\left(p_{1}, \ldots, p_{4}\right) \mid\left(p_{1}, \ldots, p_{4}\right) \text { satisfies }(6)\right\} \leq \frac{2 \cdot\left\{\left(2^{n}\right) !\right\}^{4}}{2^{n}-1} .
$$


Case $x_{L R}^{(i)} \neq x_{L R}^{(j)}, x_{R L}^{(i)}=x_{R L}^{(j)}$, and $x_{R R}^{(i)}=x_{R R}^{(j)}$. For any $p_{1}$ and any $p_{2},(12)$ is satisfied. Therefore, for any $p_{1}$, any $p_{2}$, and any $p_{3}$, the number of $p_{4}$ which satisfies (13) (which is equivalent to (14)) is at most $\frac{\left(2^{n}\right) !}{2^{n}-1}$. Therefore we have

$$
\#\left\{\left(p_{1}, p_{2}, p_{3}, p_{4}\right) \mid\left(p_{1}, p_{2}, p_{3}, p_{4}\right) \text { satisfies }(6)\right\} \leq \frac{\left\{\left(2^{n}\right) !\right\}^{4}}{2^{n}-1} .
$$

Case $x_{L L}^{(i)} \neq x_{L L}^{(j)}, x_{L R}^{(i)}=x_{L R}^{(j)}, x_{R L}^{(i)}=x_{R L}^{(j)}$, and $x_{R R}^{(i)}=x_{R R}^{(j)}$. There exists no $p_{1}, p_{2}, p_{3}$, and $p_{4}$ that satisfies (6). Therefore we have

$$
\#\left\{\left(p_{1}, p_{2}, p_{3}, p_{4}\right) \mid\left(p_{1}, p_{2}, p_{3}, p_{4}\right) \text { satisfies }(6)\right\}=0 \text {. }
$$

Completing the proof. By taking the maximum of (16), (19), (20) and (21),

$$
\#\left\{\left(p_{1}, \ldots, p_{4}\right) \mid\left(p_{1}, \ldots, p_{4}\right) \text { satisfies }(6)\right\} \leq \frac{4 \cdot\left\{\left(2^{n}\right) !\right\}^{4}}{2^{n}-1} .
$$

for any case.

From (22) and since we have $\left(\begin{array}{l}q \\ 2\end{array}\right)$ choice of $i$ and $j$ the lemma follows.

Q.E.D.

Next we show the following lemma.

Lemma 4.3 For any fixed possible view $v=\left\langle\left(x^{(1)}, y^{(1)}\right), \ldots,\left(x^{(q)}, y^{(q)}\right)\right\rangle$ which satisfies the condition of Lemma 3.1, the number of $\left(p_{1}, p_{2}, p_{3}, p_{4}\right)$ such that

$$
O_{9}^{(i)}=O_{9}^{(j)} \text { for } 1 \leq \exists i<\exists j \leq q
$$

is at most $\frac{1}{2} \cdot \frac{q(q-1)}{2^{n}-1} \cdot\left\{\left(2^{n}\right) !\right\}^{4}$.

Proof. First, we fix $i$ and $j$ such that $1 \leq i<j \leq q$, and consider $O_{9}^{(i)}=O_{9}^{(j)}$. Now observe that for any $p_{1}$ and $p_{2}, O_{9}^{(i)}=O_{9}^{(j)}$ is equivalent to the following condition:

$$
p_{3}\left(I_{3}^{(i)}\right) \oplus x_{L L}^{(i)} \oplus y_{L L}^{(i)} \oplus x_{L R}^{(i)} \oplus y_{L R}^{(i)}=p_{3}\left(I_{3}^{(j)}\right) \oplus x_{L L}^{(j)} \oplus y_{L L}^{(j)} \oplus x_{L R}^{(j)} \oplus y_{L R}^{(j)}
$$

Then the number of $p_{3}$ which satisfies (24) is at most $\frac{\left(2^{n}\right) !}{2^{n}-1}$, since $x_{L L}^{(i)} \oplus y_{L L}^{(i)} \oplus x_{L R}^{(i)} \oplus y_{L R}^{(i)} \neq$ $x_{L L}^{(j)} \oplus y_{L L}^{(j)} \oplus x_{L R}^{(j)} \oplus y_{L R}^{(j)}$.

Therefore, we have

$$
\#\left\{\left(p_{1}, \ldots, p_{4}\right) \mid\left(p_{1}, \ldots, p_{4}\right) \text { satisfies }(6)\right\} \leq \frac{\left\{\left(2^{n}\right) !\right\}^{4}}{2^{n}-1}
$$

and since we have $\left(\begin{array}{l}q \\ 2\end{array}\right)$ choice of $i$ and $j$ the lemma follows.

Q.E.D.

We now prove Lemma 3.1.

Proof of Lemma 3.1. Initially, $x^{(1)}, \ldots, x^{(q)}, y^{(1)}, \ldots, y^{(q)}$ are fixed. See Fig. 4. 


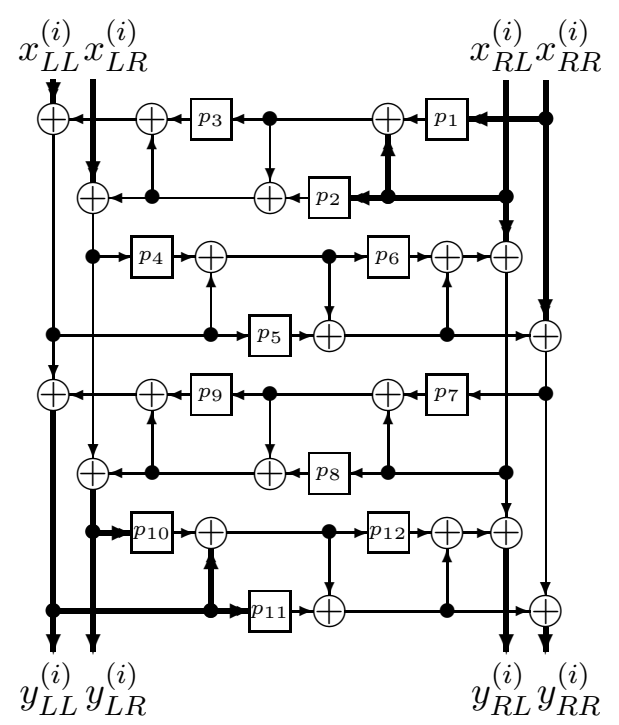

Fig. 4. $x^{(i)}$ and $y^{(i)}$ are fixed.

Number of $\left(p_{1}, \ldots, p_{4}\right)$. From Lemma 4.2 and 4.3 , the number of $\left(p_{1}, \ldots, p_{4}\right)$ such that:

- $I_{6}^{(i)} \neq I_{6}^{(j)}, I_{6}^{(i)} \oplus x_{R R}^{(i)} \neq I_{6}^{(j)} \oplus x_{R R}^{(j)}$, and $O_{9}^{(i)} \neq O_{9}^{(j)}$ for $1 \leq \forall i<\forall j \leq q$,

is at least $\left\{\left(2^{n}\right) !\right\}^{4}-\frac{1}{2} \cdot \frac{q(q-1)}{2^{n}-1} \cdot\left\{\left(2^{n}\right) !\right\}^{4}-\frac{2 q(q-1)}{2^{n}-1} \cdot\left\{\left(2^{n}\right) !\right\}^{4}$. Fix any $\left(p_{1}, \ldots, p_{4}\right)$ which satisfy these three conditions. See Fig. 5 .

Number of $p_{5}$. For any fixed $i$ and $j$ such that $1 \leq i<j \leq q$, the number of $p_{5}$ such that $p_{5}\left(I_{5}^{(i)}\right) \oplus I_{6}^{(i)} \oplus x_{R R}^{(i)}=p_{5}\left(I_{5}^{(j)}\right) \oplus I_{6}^{(j)} \oplus x_{R R}^{(j)}$, which is equivalent to $I_{7}^{(i)}=I_{7}^{(j)}$, is at most $\frac{\left\{\left(2^{n}\right) !\right\}^{4}}{2^{n}-1}$ since $I_{6}^{(i)} \oplus x_{R R}^{(i)} \neq I_{6}^{(j)} \oplus x_{R R}^{(j)}$. Then the number of $p_{5}$ such that

- $I_{7}^{(i)} \neq I_{7}^{(j)}$ for $1 \leq \forall i<\forall j \leq q$

is at least $\left(2^{n}\right) !-\frac{1}{2} \cdot \frac{q(q-1)}{2^{n}-1} \cdot\left(2^{n}\right)$ !. Fix any such $p_{5}$. See Fig. 6 .

Number of $p_{6}$. For any fixed $i$ and $j$ such that $1 \leq i<j \leq q$, the number of $p_{6}$ which satisfies $p_{6}\left(I_{6}^{(i)}\right) \oplus I_{6}^{(i)} \oplus O_{5}^{(i)} \oplus x_{R L}^{(i)}=p_{6}\left(I_{6}^{(j)}\right) \oplus I_{6}^{(j)} \oplus O_{5}^{(j)} \oplus x_{R L}^{(j)}$, which is equivalent to $I_{8}^{(i)}=I_{8}^{(j)}$, is at most $\frac{\left(2^{n}\right) !}{2^{n}-1}$, since $I_{6}^{(i)} \neq I_{6}^{(j)}$.

Similarly, the number of $p_{6}$ which satisfies $p_{6}\left(I_{6}^{(i)}\right) \oplus I_{6}^{(i)} \oplus O_{5}^{(i)} \oplus x_{R L}^{(i)} \oplus I_{7}^{(i)} \oplus y_{R L}^{(i)} \oplus y_{R R}^{(i)}=$ $p_{6}\left(I_{6}^{(j)}\right) \oplus I_{6}^{(j)} \oplus O_{5}^{(j)} \oplus x_{R L}^{(j)} \oplus I_{7}^{(j)} \oplus y_{R L}^{(j)} \oplus y_{R R}^{(j)}$, which is equivalent to $O_{12}^{(i)}=O_{12}^{(j)}$, is at most $\frac{\left(2^{n}\right) !}{2^{n}-1}$, since $I_{6}^{(i)} \neq I_{6}^{(j)}$.

Then, the number of $p_{6}$ which satisfies:

- $I_{8}^{(i)} \neq I_{8}^{(j)}$ and $O_{12}^{(i)} \neq O_{12}^{(j)}$ for $1 \leq \forall i<\forall j \leq q$,

is at least $\left(2^{n}\right) !-\frac{q(q-1)}{2^{n}-1} \cdot\left(2^{n}\right)$ !. Fix any $p_{6}$ which satisfy the above two conditions. See Fig. 7 . 


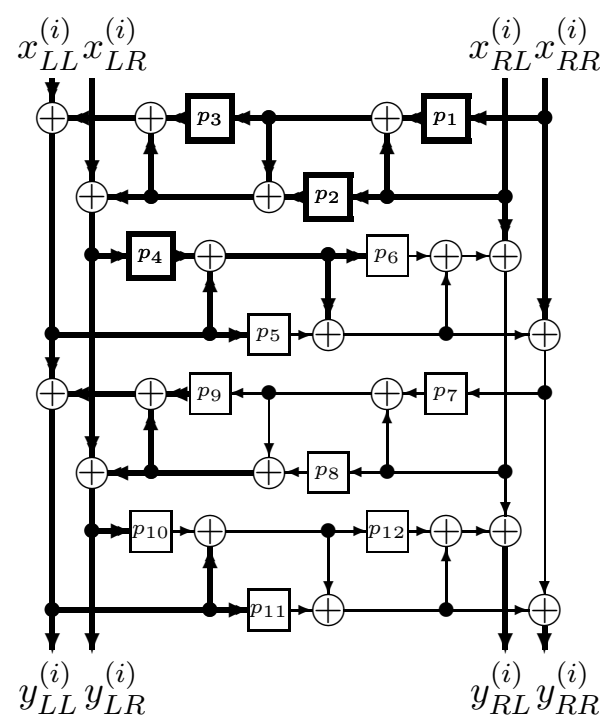

Fig. 5. $p_{1}, \ldots, p_{4}$ are fixed.

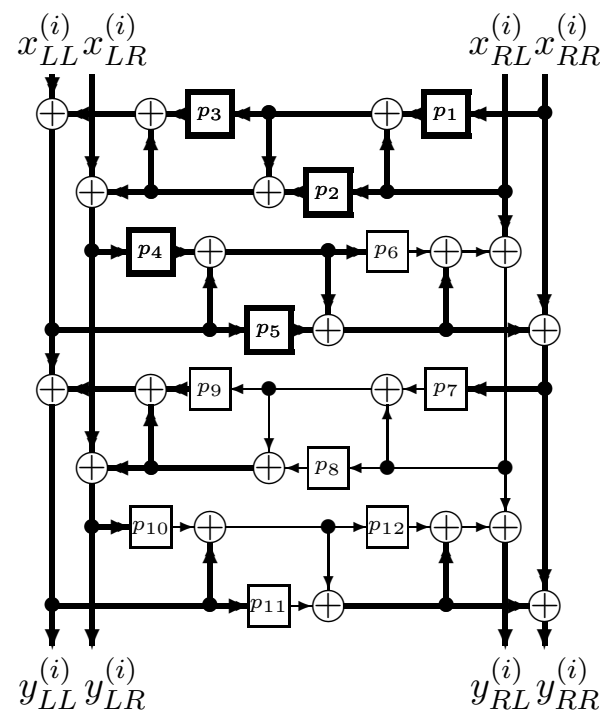

Fig. 6. $p_{5}$ is fixed. 


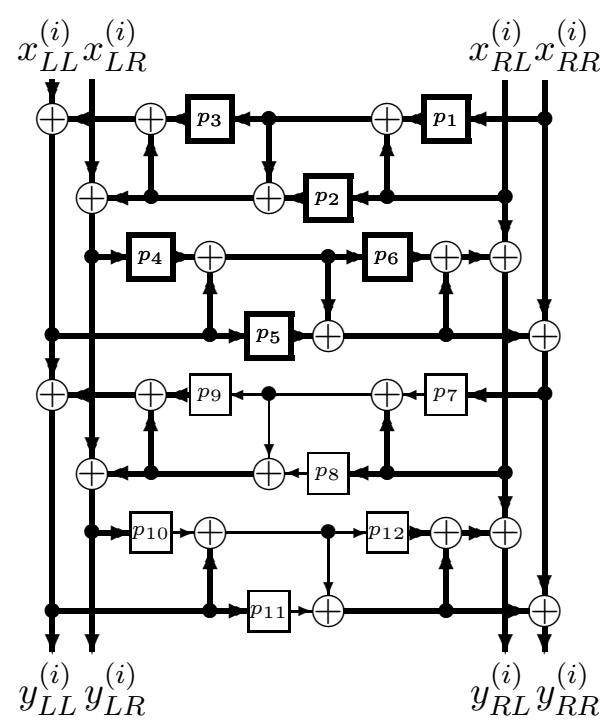

Fig. 7. $p_{6}$ is fixed.

Number of $\left(p_{7}, \ldots, p_{12}\right)$. Now $p_{1}, \ldots, p_{6}$ are fixed in such a way that $\left\{I_{7}^{(i)}\right\}_{1 \leq i \leq q}$ are distinct, $\left\{I_{8}^{(i)}\right\}_{1 \leq i \leq q}$ are distinct, $\left\{O_{9}^{(i)}\right\}_{1 \leq i \leq q}$ are distinct and $\left\{O_{12}^{(i)}\right\}_{1 \leq i \leq q}$ are distinct. We know from our condition that $\left\{I_{10}^{(i)}\right\}_{1 \leq i \leq q}$ are distinct and $\left\{I_{11}^{(i)}\right\}_{1 \leq i \leq q}$ are distinct.

Then we have at least $\left(1-\frac{q(q-1)}{2^{n}-1}\right) \cdot\left(2^{n}\right) ! \cdot\left\{\left(2^{n}-q\right) !\right\}^{2}$ choice of $\left(p_{7}, p_{8}, p_{9}\right)$ by applying Lemma 4.1. That is, $X_{L}^{(i)}, X_{R}^{(i)}, Y_{L}^{(i)} \oplus Y_{R}^{(i)}, P_{1}, P_{2}$ and $P_{3}$ in Lemma 4.1 correspond to $I_{7}^{(i)}$, $I_{8}^{(i)}, O_{9}^{(i)}, p_{7}, p_{8}$ and $p_{9}$ respectively.

Similarly, from Lemma 4.1 we have at least $\left(1-\frac{q(q-1)}{2^{n}-1}\right) \cdot\left(2^{n}\right) ! \cdot\left\{\left(2^{n}-q\right) !\right\}^{2}$ choice of $\left(p_{10}, p_{11}, p_{12}\right)$. Note that $X_{L}^{(i)}, X_{R}^{(i)}, Y_{L}^{(i)} \oplus Y_{R}^{(i)}, P_{1}, P_{2}$ and $P_{3}$ in Lemma 4.1 correspond to $I_{10}^{(i)}$, $I_{11}^{(i)}, O_{12}^{(i)}, p_{10}, p_{11}$ and $p_{12}$ respectively.

Completing the proof. To summarize, we have:

- at least $\left(1-\frac{5}{2} \cdot \frac{q(q-1)}{2^{n}-1}\right) \cdot\left\{\left(2^{n}\right) !\right\}^{4}$ choice of $p_{1}, \ldots, p_{4}$,

- at least $\left(2^{n}\right) !-\frac{1}{2} \cdot \frac{q(q-1)}{2^{n}-1} \cdot\left(2^{n}\right)$ ! choice of $p_{5}$ when $p_{1}, \ldots, p_{4}$ are fixed,

- at least $\left(2^{n}\right) !-\frac{q(q-1)}{2^{n}-1} \cdot\left(2^{n}\right)$ ! choice of $p_{6}$ when $p_{1}, \ldots, p_{5}$ are fixed, and

- at least $\left(1-\frac{q(q-1)}{2^{n}-1}\right)^{2} \cdot\left\{\left(2^{n}\right) !\right\}^{2} \cdot\left\{\left(2^{n}-q\right) !\right\}^{4}$ choice of $p_{7} \ldots, p_{12}$ when $p_{1}, \ldots, p_{6}$ are fixed.

Then, the number of $\left(p_{1}, \ldots, p_{12}\right)$ which satisfy $(2)$ is at least

$$
\begin{aligned}
& \left(1-\frac{5}{2} \cdot \frac{q(q-1)}{2^{n}-1}\right) \cdot\left(1-\frac{1}{2} \cdot \frac{q(q-1)}{2^{n}-1}\right) \cdot\left(1-\frac{q(q-1)}{2^{n}-1}\right)^{3} \cdot\left\{\left(2^{n}\right) !\right\}^{8} \cdot\left\{\left(2^{n}-q\right) !\right\}^{4} \\
& \geq\left(1-\frac{6 q(q-1)}{2^{n}-1}\right) \cdot\left\{\left(2^{n}\right) !\right\}^{8} \cdot\left\{\left(2^{n}-q\right) !\right\}^{4} .
\end{aligned}
$$

This concludes the proof of the lemma.

Q.E.D. 


\subsection{Proof of Lemma 3.2}

For any fixed $i$ and $j$ such that $1 \leq i<j \leq q$, the number of $\left\{y^{(i)}\right\}_{1 \leq i \leq q}$ such that $y_{L L}^{(i)}=y_{L L}^{(j)}$ is at most $\frac{2^{3 n}-1}{2^{4 n}-(j-1)} \cdot \frac{\left(2^{4 n}\right) !}{\left(2^{4 n}-q\right) !} \leq \frac{2^{3 n}-1}{2^{4 n}-(q-1)} \cdot \frac{\left(2^{4 n}\right) !}{\left(2^{4 n}-q\right) !}$, since we have: $\left(2^{4 n}\right)\left(2^{4 n}-1\right) \cdots\left(2^{4 n}-(j-2)\right)$ choice of $y^{(1)}, \ldots, y^{(j-1)}$, which uniquely determines $y_{L L}^{(j)}=y_{L L}^{(i)}$; at most $2^{3 n}-1$ choice of $y_{L R}^{(j)}, y_{R L}^{(j)}, y_{R R}^{(j)}$; and $\left(2^{4 n}-j\right)\left(2^{4 n}-j-1\right) \cdots\left(2^{4 n}-(q-1)\right)$ choice of $y^{(j+1)}, \ldots, y^{(q)}$.

Similarly, for any fixed $i$ and $j$ such that $1 \leq i<j \leq q$, the number of $\left\{y^{(i)}\right\}_{1 \leq i \leq q}$ such that $y_{L R}^{(i)}=y_{L R}^{(j)}$ is at most $\frac{2^{3 n}-1}{2^{4 n}-(q-1)} \cdot \frac{\left(2^{4 n}\right) !}{\left(2^{4 n}-q\right) !}$.

Next, for any fixed $i$ and $j$ such that $1 \leq i<j \leq q$, the number of $\left\{y^{(i)}\right\}_{1 \leq i \leq q}$ such that $x_{L L}^{(i)} \oplus x_{L R}^{(i)} \oplus y_{L L}^{(i)} \oplus y_{L R}^{(i)}=x_{L L}^{(j)} \oplus x_{L R}^{(j)} \oplus y_{L L}^{(j)} \oplus y_{L R}^{(j)}$ is at most $\frac{2^{3 n}}{2^{4 n}-(j-1)} \cdot \frac{\left(2^{4 n}\right) !}{\left(2^{4 n}-q\right) !} \leq \frac{2^{3 n}}{2^{4 n}-(q-1)} \cdot \frac{\left(2^{4 n}\right) !}{\left(2^{4 n}-q\right) !}$, since we have: $\left(2^{4 n}\right)\left(2^{4 n}-1\right) \cdots\left(2^{4 n}-(j-2)\right)$ choice of $y^{(1)}, \ldots, y^{(j-1)} ; 2^{n}$ choice of $y_{L R}^{(j)}$, which uniquely determines $y_{L L}^{(j)}=x_{L L}^{(i)} \oplus x_{L R}^{(i)} \oplus y_{L L}^{(i)} \oplus y_{L R}^{(i)} \oplus x_{L L}^{(j)} \oplus x_{L R}^{(j)} \oplus y_{L R}^{(j)}$; at most $2^{2 n}$ choice of $y_{R L}^{(j)}, y_{R R}^{(j)}$; and $\left(2^{4 n}-j\right)\left(2^{4 n}-j-1\right) \cdots\left(2^{4 n}-(q-1)\right)$ choice of $y^{(j+1)}, \ldots, y^{(q)}$.

Therefore, the number of $y^{(1)}, \ldots, y^{(q)}$ such that

- $y_{L L}^{(i)}=y_{L L}^{(j)}$ for $1 \leq \exists i<\exists j \leq q$,

- $y_{L R}^{(i)}=y_{L R}^{(j)}$ for $1 \leq \exists i<\exists j \leq q$, or

- $x_{L L}^{(i)} \oplus x_{L R}^{(i)} \oplus y_{L L}^{(i)} \oplus y_{L R}^{(i)}=x_{L L}^{(j)} \oplus x_{L R}^{(j)} \oplus y_{L L}^{(j)} \oplus y_{L R}^{(j)}$ for $1 \leq \exists i<\exists j \leq q$

is at most $\left(\begin{array}{l}q \\ 2\end{array}\right) \cdot \frac{3 \cdot 2^{3 n}-2}{2^{4 n}-(q-1)} \cdot \frac{\left\{\left(2^{4 n}\right) !\right\}}{\left\{\left(2^{4 n}-q\right) !\right\}}$, which is at most $\frac{3}{2} \cdot \frac{q(q-1)}{2^{n}-1} \cdot \frac{\left\{\left(2^{4 n}\right) !\right\}}{\left\{\left(2^{4 n}-q\right) !\right\}}$.

Q.E.D.

\section{A six round KASUMI type permutation is super-pseudorandom}

Theorem 5.1 For $1 \leq i \leq 18$, let $p_{i} \in P_{n}$ be a random permutation. Let $\psi=\psi\left(p_{1}, \ldots, p_{18}\right)$ be a six round KASUMI type permutation, $R \in P_{4 n}$ be a random permutation, and $\Psi \stackrel{\text { def }}{=}\{\psi \mid \psi=$ $\psi\left(p_{1}, \ldots, p_{18}\right), p_{i} \in P_{n}$ for $\left.1 \leq i \leq 18\right\}$.

Then for any adversary $\mathcal{A}$ that makes at most q queries in total,

$$
\operatorname{Adv}_{\Psi}^{\text {sprp }}(\mathcal{A}) \leq \frac{9 q(q-1)}{2^{n}-1}
$$

Proof. Let $\mathcal{O}$ be either $R$ or $\psi$. The adversary $\mathcal{A}$ has oracle access to $\mathcal{O}$ and $\mathcal{O}^{-1}$.

There are two types of queries $\mathcal{A}$ can make: either $(+, x)$ or $(-, y)$. For the $i$-th query $\mathcal{A}$ makes to $\mathcal{O}$ or $\mathcal{O}^{-1}$, define the query-answer pair $\left(x^{(i)}, y^{(i)}\right) \in\{0,1\}^{4 n} \times\{0,1\}^{4 n}$, where either $\mathcal{A}$ 's query was $\left(+, x^{(i)}\right)$ and the answer it got was $y^{(i)}=\mathcal{O}\left(x^{(i)}\right)$ or $\mathcal{A}$ 's query was $\left(-, y^{(i)}\right)$ and the answer it got was $x^{(i)}=\mathcal{O}^{-1}\left(y^{(i)}\right)$. Define view $v$ of $\mathcal{A}$ as $v=\left\langle\left(x^{(1)}, y^{(1)}\right), \ldots,\left(x^{(q)}, y^{(q)}\right)\right\rangle$.

Since $\mathcal{A}$ has unbounded computational power, $\mathcal{A}$ can be assumed to be deterministic. This implies that there exists a function $\mathcal{C}_{\mathcal{A}}$ such that

$$
\left\{\begin{array}{l}
\mathcal{C}_{\mathcal{A}}\left(x^{(1)}, y^{(1)}, \ldots, x^{(i-1)}, y^{(i-1)}\right)=\text { either }\left(+, x^{(i)}\right) \text { or }\left(-, y^{(i)}\right) \text { for } 1 \leq i \leq q \text { and } \\
\mathcal{C}_{\mathcal{A}}(v)=\mathcal{A} \text { 's final output. }
\end{array}\right.
$$

Let $\boldsymbol{v}_{\text {one }} \stackrel{\text { def }}{=}\left\{v \mid \mathcal{C}_{\mathcal{A}}(v)=1\right\}$ and $N_{\text {one }} \stackrel{\text { def }}{=} \# \boldsymbol{v}_{\text {one }}$. 
Evaluation of $p_{R}$. We first evaluate $p_{R} \stackrel{\text { def }}{=} \operatorname{Pr}\left(R \stackrel{R}{\leftarrow} P_{4 n}: \mathcal{A}^{R, R^{-1}}=1\right)$. We have $p_{R}=$ $N_{\text {one }} \cdot \frac{\left(2^{4 n}-q\right) !}{\left(2^{4 n}\right) !}$ as was done in the proof of Theorem 3.1

Evaluation of $p_{\psi}$. We evaluate $p_{\psi} \stackrel{\text { def }}{=} \operatorname{Pr}\left(\psi \stackrel{R}{\leftarrow} \Psi: \mathcal{A}^{\psi, \psi^{-1}}=1\right)$. Note that " $\psi \stackrel{R}{\leftarrow} \Psi$ " is equivalent to " $p_{i} \stackrel{R}{\leftarrow} P_{n}$ for $1 \leq i \leq 18$ and then let $\psi \leftarrow \psi\left(p_{1}, \ldots, p_{18}\right)$." We have $p_{\psi}=$ $\frac{\#\left\{\left(p_{1}, \ldots, p_{18}\right) \mid \mathcal{A}^{\psi, \psi^{-1}}=1\right\}}{\left\{\left(2^{n}\right) !\right\}^{18}}$.

We have the following lemma. A proof of this lemma is given in Section 6 .

Lemma 5.1 (Main Lemma for $\psi\left(p_{1}, \ldots, p_{18}\right)$ ) For any fixed possible view

$$
v=\left\langle\left(x^{(1)}, y^{(1)}\right), \ldots,\left(x^{(q)}, y^{(q)}\right)\right\rangle,
$$

the number of $\left(p_{1}, \ldots, p_{18}\right)$ such that

$$
\psi\left(x^{(i)}\right)=y^{(i)} \text { for } 1 \leq \forall i \leq q
$$

is at least $\left(1-\frac{9 q(q-1)}{2^{n}-1}\right) \cdot\left\{\left(2^{n}\right) !\right\}^{14} \cdot\left\{\left(2^{n}-q\right) !\right\}^{4}$.

Then from Lemma 5.1, we have

$$
\begin{aligned}
p_{\psi} & =\sum_{v \in \boldsymbol{v}_{\text {one }}} \frac{\#\left\{\left(p_{1}, \ldots, p_{18}\right) \mid\left(p_{1}, \ldots, p_{18}\right) \text { satisfying }(25)\right\}}{\left\{\left(2^{n}\right) !\right\}^{18}} \\
& \geq \sum_{v \in \boldsymbol{v}_{\text {one }}}\left(1-\frac{9 q(q-1)}{2^{n}-1}\right) \cdot \frac{\left\{\left(2^{n}-q\right) !\right\}^{4}}{\left\{\left(2^{n}\right) !\right\}^{4}} \\
& \geq N_{\text {one }} \cdot\left(1-\frac{9 q(q-1)}{2^{n}-1}\right) \cdot \frac{\left\{\left(2^{n}-q\right) !\right\}^{4}}{\left\{\left(2^{n}\right) !\right\}^{4}} \\
& =p_{R} \cdot\left(1-\frac{9 q(q-1)}{2^{n}-1}\right) \cdot \frac{\left\{\left(2^{n}-q\right) !\right\}^{4}}{\left\{\left(2^{n}\right) !\right\}^{4}} \cdot \frac{\left\{\left(2^{4 n}\right) !\right\}}{\left\{\left(2^{4 n}-q\right) !\right\}} .
\end{aligned}
$$

Since $\frac{\left\{\left(2^{n}-q\right) !\right\}^{4}}{\left\{\left(2^{n}\right) !\right\}^{4}} \cdot \frac{\left\{\left(2^{4 n}\right) !\right\}}{\left\{\left(2^{4 n}-q\right) !\right\}} \geq 1, p_{\psi} \geq p_{R} \cdot\left(1-\frac{9 q(q-1)}{2^{n}-1}\right) \geq p_{R}-\frac{9 q(q-1)}{2^{n}-1}$. Applying the same argument to $1-p_{\psi}$ and $1-p_{R}$ yields that $1-p_{\psi} \geq 1-p_{R}-\frac{9 q(q-1)}{2^{n}-1}$ and we have $\left|p_{\psi}-p_{R}\right| \leq \frac{9 q(q-1)}{2^{n}-1}$.

From Theorem 5.1, it is straightforward to show that $\psi=\psi\left(p_{1}, \ldots, p_{18}\right)$ is super-pseudorandom even if each $p_{i}$ is a pseudorandom permutation. Note that we do not need the super-pseudorandomness of $p_{i}$ to derive this result, since KASUMI type permutation does not use $p_{i}^{-1}$ in both encryption and decryption. That is, we can "simulate" both $\psi$ and $\psi^{-1}$ without using $p_{i}^{-1}$.

\section{Proof of Lemma 5.1}

For $1 \leq i \leq q$ and $1 \leq j \leq 18$, let $I_{j}^{(i)}$ denote the input to $p_{i}$ when the input to $\phi$ is $x^{(i)}$ and the output is $y^{(i)}$. Similarly, let $O_{j}^{(i)}$ denote the output of $p_{i}$ when the input to $\phi$ is $x^{(i)}$ and the output is $y^{(i)}$.

Initially, $x^{(1)}, \ldots, x^{(q)}, y^{(1)}, \ldots, y^{(q)}$ are fixed. See Fig. 8 . 


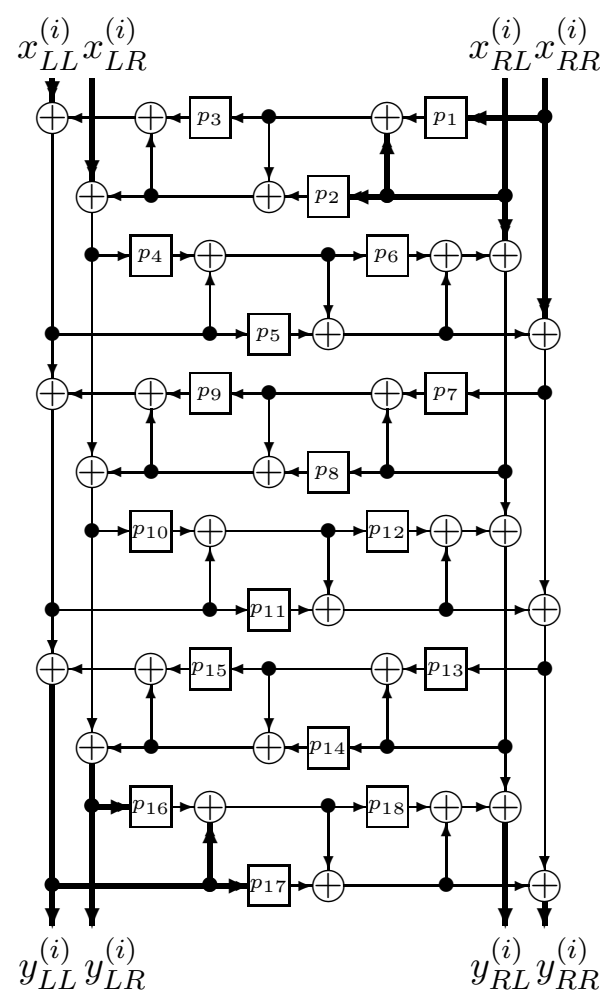

Fig. 8. $x^{(i)}$ and $y^{(i)}$ are fixed.

Number of $\left(p_{1}, \ldots, p_{4}\right)$. From Lemma 4.2 , the number of $\left(p_{1}, \ldots, p_{4}\right)$ such that:

- $I_{6}^{(i)} \neq I_{6}^{(j)}$, and $I_{6}^{(i)} \oplus x_{R R}^{(i)} \neq I_{6}^{(j)} \oplus x_{R R}^{(j)}$ for $1 \leq \forall i<\forall j \leq q$

is at least $\left\{\left(2^{n}\right) !\right\}^{4}-\frac{2 q(q-1)}{2^{n}-1} \cdot\left\{\left(2^{n}\right) !\right\}^{4}$. Note that Lemma 4.2 holds for any possible view, and it is irrelevant from the condition on $y^{(i)}$ in Lemma 3.1. Fix $\left(p_{1}, \ldots, p_{4}\right)$ which satisfy these two conditions arbitrarily.

Number of $\left(p_{13}, p_{16}, p_{17}, p_{18}\right)$. From Lemma 4.2 , the number of $\left(p_{13}, p_{16}, p_{17}, p_{18}\right)$ such that: - $I_{15}^{(i)} \neq I_{15}^{(j)}$, and $I_{15}^{(i)} \oplus y_{L R}^{(i)} \neq I_{15}^{(j)} \oplus y_{L R}^{(j)}$ for $1 \leq \forall i<\forall j \leq q$ is at least $\left\{\left(2^{n}\right) !\right\}^{4}-\frac{2 q(q-1)}{2^{n}-1} \cdot\left\{\left(2^{n}\right) !\right\}^{4}$. We have used the symmetry of KASUMI type permutation. That is, $x_{L L}^{(i)}, x_{L R}^{(i)}, x_{R L}^{(i)}, x_{R R}^{(i)}, p_{1}, p_{2}, p_{3}, p_{4}$ and $I_{6}^{(i)}$ in Lemma 4.2 corresponds to $y_{R L}^{(i)}, y_{R R}^{(i)}$, $y_{L L}^{(i)}, y_{L R}^{(i)}, p_{16}, p_{17}, p_{18}, p_{13}$ and $I_{15}^{(i)}$ respectively. Fix $\left(p_{13}, p_{16}, p_{17}, p_{18}\right)$ which satisfy these two conditions arbitrarily. See Fig. 9.

Number of $p_{5}$. For any fixed $i$ and $j$ such that $1 \leq i<j \leq q$, the number of $p_{5}$ such that $p_{5}\left(I_{5}^{(i)}\right) \oplus I_{6}^{(i)} \oplus x_{R R}^{(i)}=p_{5}\left(I_{5}^{(j)}\right) \oplus I_{6}^{(j)} \oplus x_{R R}^{(j)}$, which is equivalent to $I_{7}^{(i)}=I_{7}^{(j)}$, is at most $\frac{\left\{\left(2^{n}\right) !\right\}^{4}}{2^{n}-1}$ since $I_{6}^{(i)} \oplus x_{R R}^{(i)} \neq I_{6}^{(j)} \oplus x_{R R}^{(j)}$. Then the number of $p_{5}$ such that

- $I_{7}^{(i)} \neq I_{7}^{(j)}$ for $1 \leq \forall i<\forall j \leq q$

is at least $\left(2^{n}\right) !-\frac{1}{2} \cdot \frac{q(q-1)}{2^{n}-1} \cdot\left(2^{n}\right)$ !. Fix any $p_{5}$ which satisfy the above condition. 


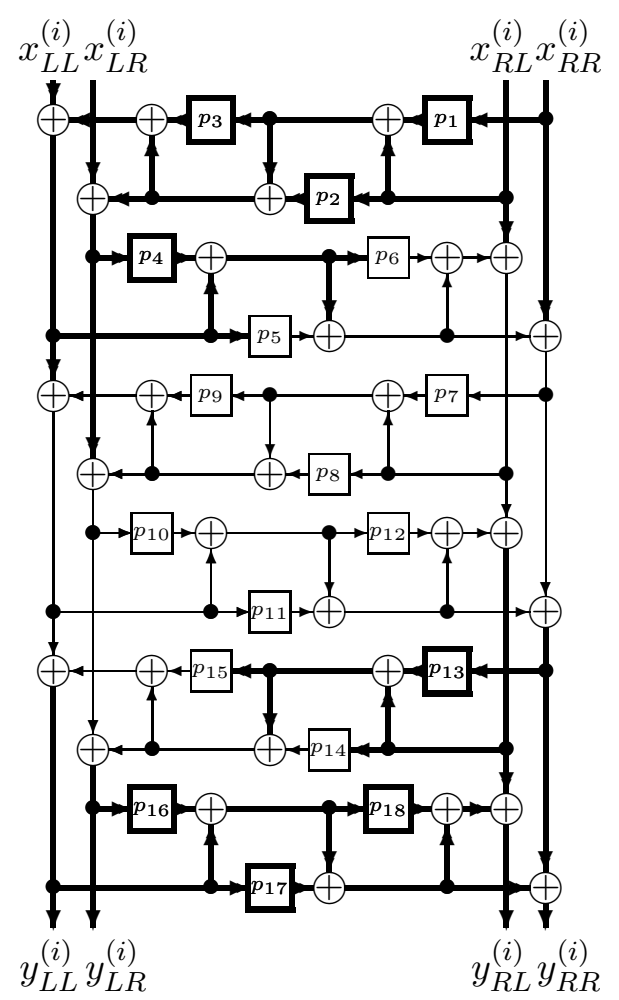

Fig. 9. $p_{1}, \ldots, p_{4}, p_{13}, p_{16}, p_{17}, p_{18}$ are fixed.

Number of $p_{14}$. Similar to the case $p_{5}$, for any fixed $i$ and $j$ such that $1 \leq i<j \leq q$, the number of $p_{14}$ such that $p_{14}\left(I_{14}^{(i)}\right) \oplus I_{15}^{(i)} \oplus y_{L R}^{(i)}=p_{14}\left(I_{14}^{(j)}\right) \oplus I_{15}^{(j)} \oplus y_{L R}^{(j)}$, which is equivalent to $I_{10}^{(i)}=I_{10}^{(j)}$, is at most $\frac{\left\{\left(2^{n}\right) !\right\}^{4}}{2^{n}-1}$ since $I_{15}^{(i)} \oplus y_{L R}^{(i)} \neq I_{15}^{(j)} \oplus y_{L R}^{(j)}$. Then the number of $p_{14}$ such that

- $I_{10}^{(i)} \neq I_{10}^{(j)}$ for $1 \leq \forall i<\forall j \leq q$

is at least $\left(2^{n}\right) !-\frac{1}{2} \cdot \frac{q(q-1)}{2^{n}-1} \cdot\left(2^{n}\right)$ !. Fix any $p_{14}$ which satisfy the above condition. See Fig. 10 .

Number of $p_{6}$. For any fixed $i$ and $j$ such that $1 \leq i<j \leq q$, the number of $p_{6}$ which satisfies $p_{6}\left(I_{6}^{(i)}\right) \oplus I_{6}^{(i)} \oplus O_{5}^{(i)} \oplus x_{R L}^{(i)}=p_{6}\left(I_{6}^{(j)}\right) \oplus I_{6}^{(j)} \oplus O_{5}^{(j)} \oplus x_{R L}^{(j)}$, which is equivalent to $I_{8}^{(j)}=I_{8}^{(j)}$, is at most $\frac{\left(2^{n}\right) !}{2^{n}-1}$, since $I_{6}^{(i)} \neq I_{6}^{(j)}$.

Similarly, the number of $p_{6}$ which satisfies $p_{6}\left(I_{6}^{(i)}\right) \oplus I_{6}^{(i)} \oplus O_{5}^{(i)} \oplus x_{R L}^{(i)} \oplus I_{14}^{(i)} \oplus I_{7}^{(i)} \oplus I_{13}^{(i)}=$ $p_{6}\left(I_{6}^{(j)}\right) \oplus I_{6}^{(j)} \oplus O_{5}^{(j)} \oplus x_{R L}^{(j)} \oplus I_{14}^{(j)} \oplus I_{7}^{(j)} \oplus I_{13}^{(j)}$, which is equivalent to $O_{12}^{(i)}=O_{12}^{(j)}$, is at most $\frac{\left(2^{n}\right) !}{2^{n}-1}$, since $I_{6}^{(i)} \neq I_{6}^{(j)}$.

Then, the number of $p_{6}$ which satisfies

- $I_{8}^{(i)} \neq I_{8}^{(j)}$ and $O_{12}^{(i)} \neq O_{12}^{(j)}$ for $1 \leq \forall i<\forall j \leq q$

is at least $\left(2^{n}\right) !-\frac{q(q-1)}{2^{n}-1} \cdot\left(2^{n}\right)$ !. Fix any $p_{6}$ which satisfy the above condition. 


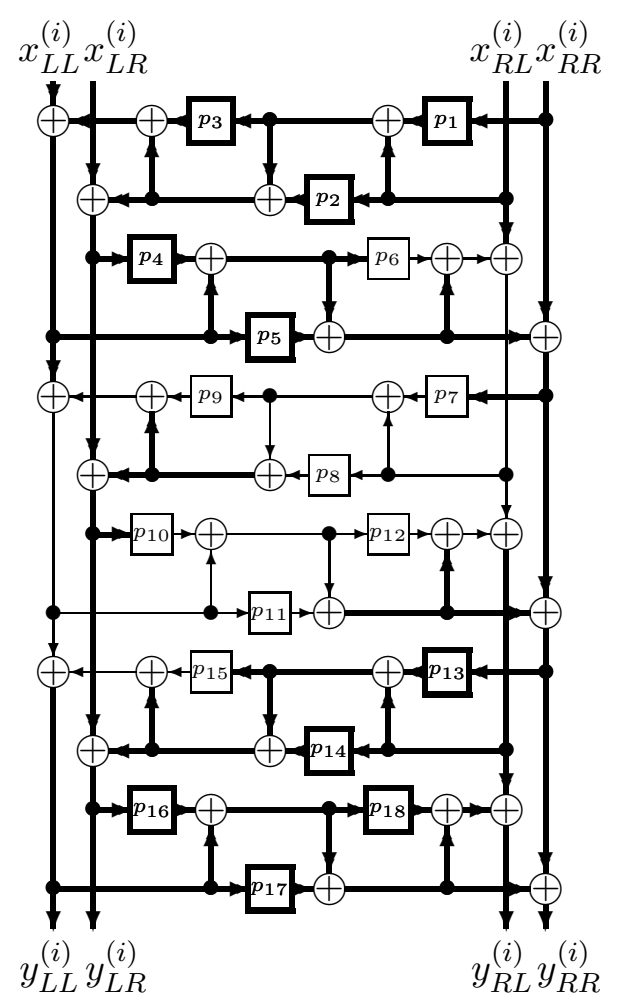

Fig. 10. $p_{5}$ and $p_{14}$ are fixed.

Number of $p_{15}$. For any fixed $i$ and $j$ such that $1 \leq i<j \leq q$, the number of $p_{15}$ which satisfies $p_{15}\left(I_{15}^{(i)}\right) \oplus I_{15}^{(i)} \oplus O_{14}^{(i)} \oplus y_{L L}^{(i)}=p_{15}\left(I_{15}^{(j)}\right) \oplus I_{15}^{(j)} \oplus O_{14}^{(j)} \oplus y_{L L}^{(j)}$, which is equivalent to $I_{11}^{(i)}=I_{11}^{(j)}$, is at most $\frac{\left(2^{n}\right) !}{2^{n}-1}$, since $I_{15}^{(i)} \neq I_{15}^{(j)}$.

Similarly, the number of $p_{15}$ which satisfies $p_{15}\left(I_{15}^{(i)}\right) \oplus I_{15}^{(i)} \oplus O_{14}^{(i)} \oplus y_{L L}^{(i)} \oplus I_{4}^{(i)} \oplus I_{10}^{(i)} \oplus I_{5}^{(i)}=$ $p_{15}\left(I_{15}^{(j)}\right) \oplus I_{15}^{(j)} \oplus O_{14}^{(j)} \oplus y_{L L}^{(i)} \oplus I_{4}^{(j)} \oplus I_{10}^{(j)} \oplus I_{5}^{(j)}$, which is equivalent to $O_{9}^{(i)}=O_{9}^{(j)}$, is at most $\frac{\left(2^{n}\right) !}{2^{n}-1}$, since $I_{15}^{(i)} \neq I_{15}^{(j)}$.

Then, the number of $p_{15}$ which satisfies

- $I_{11}^{(i)} \neq I_{11}^{(j)}$ and $O_{9}^{(i)} \neq O_{9}^{(j)}$ for $1 \leq \forall i<\forall j \leq q$

is at least $\left(2^{n}\right) !-\frac{q(q-1)}{2^{n}-1} \cdot\left(2^{n}\right)$ !. Fix any $p_{15}$ which satisfy the above condition. See Fig. 11.

Number of $\left(p_{7}, \ldots, p_{12}\right)$. Now $p_{1}, \ldots, p_{6}, p_{13}, \ldots, p_{15}$ are fixed in such a way that $\left\{I_{7}^{(i)}\right\}_{1 \leq i \leq q}$ are distinct, $\left\{I_{8}^{(i)}\right\}_{1 \leq i \leq q}$ are distinct, $\left\{O_{9}^{(i)}\right\}_{1 \leq i \leq q}$ are distinct, $\left\{I_{10}^{(i)}\right\}_{1 \leq i \leq q}$ are distinct, $\left\{I_{11}^{(i)}\right\}_{1 \leq i \leq q}$ are distinct and $\left\{O_{12}^{(i)}\right\}_{1 \leq i \leq q}$ are distinct. Then, by applying Lemma 4.1 twice, we have at least $\left(1-\frac{q(q-1)}{2^{n}-1}\right)^{2} \cdot\left\{\left(2^{n}\right) !\right\}^{2} \cdot\left\{\left(2^{n}-q\right) !\right\}^{4}$ choice of $\left(p_{7}, \ldots, p_{12}\right)$.

Completing the proof. To summarize, we have:

- at least $\left(1-\frac{2 q(q-1)}{2^{n}-1}\right)^{2} \cdot\left\{\left(2^{n}\right) !\right\}^{8}$ choice of $p_{1}, \ldots, p_{4}, p_{13}, p_{16}, p_{17}$ and $p_{18}$, 


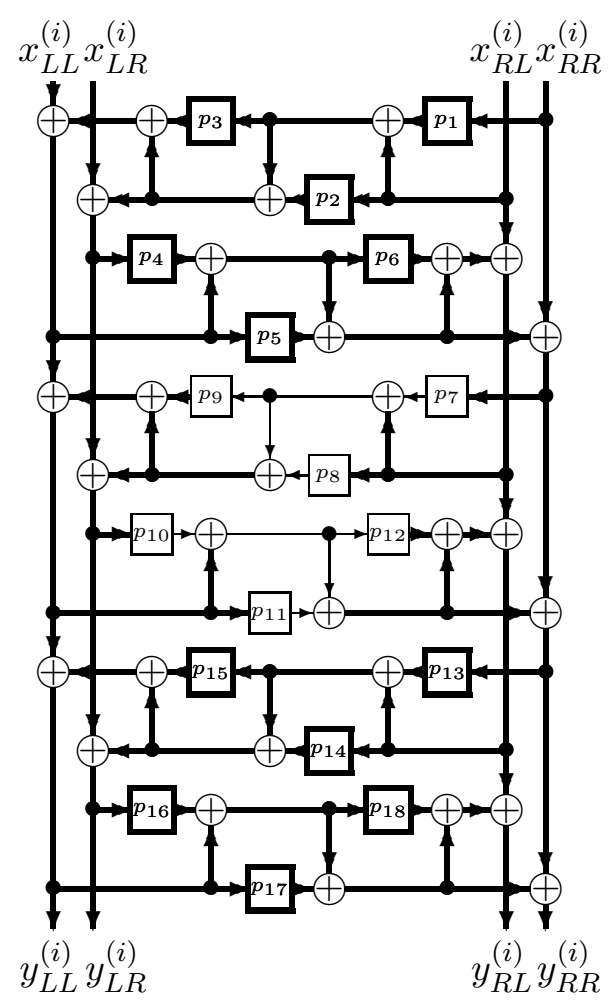

Fig. 11. $p_{6}$ and $p_{15}$ are fixed.

- at least $\left\{\left(2^{n}\right) !\right\}^{2} \cdot\left(1-\frac{1}{2} \cdot \frac{q(q-1)}{2^{n}-1}\right)^{2}$ choice of $\left(p_{5}, p_{14}\right)$ when $p_{1}, \ldots, p_{4}, p_{13}, p_{16}, p_{17}$ and $p_{18}$ are fixed,

- at least $\left\{\left(2^{n}\right) !\right\}^{2} \cdot\left(1-\frac{q(q-1)}{2^{n}-1}\right)^{2}$ choice of $\left(p_{6}, p_{15}\right)$ when $p_{1}, \ldots, p_{5}, p_{13}, p_{14}, p_{16}, p_{17}$ and $p_{18}$ are fixed,

- at least $\left(1-\frac{q(q-1)}{2^{n}-1}\right)^{2} \cdot\left\{\left(2^{n}\right) !\right\}^{2} \cdot\left\{\left(2^{n}-q\right) !\right\}^{4}$ choice of $p_{7} \ldots, p_{12}$ when $p_{1}, \ldots, p_{6}, p_{13}, \ldots, p_{18}$ are fixed.

Then the number of $\left(p_{1}, \cdots, p_{18}\right)$ which satisfy $(2)$ is at least

$$
\begin{gathered}
\left(1-\frac{2 q(q-1)}{2^{n}-1}\right)^{2} \cdot\left(1-\frac{1}{2} \cdot \frac{q(q-1)}{2^{n}-1}\right)^{2} \cdot\left(1-\frac{q(q-1)}{2^{n}-1}\right)^{4} \cdot\left\{\left(2^{n}\right) !\right\}^{14} \cdot\left\{\left(2^{n}-q\right) !\right\}^{4} \\
\geq\left(1-\frac{9 q(q-1)}{2^{n}-1}\right) \cdot\left\{\left(2^{n}\right) !\right\}^{8} \cdot\left\{\left(2^{n}-q\right) !\right\}^{4} .
\end{gathered}
$$

This concludes the proof of the lemma.

Q.E.D.

\section{Conclusion}

In this paper, we showed that a four round KASUMI type permutation is pseudorandom (Theorem 3.1 . We proved that the advantage is at most $\frac{15}{2} \cdot \frac{q(q-1)}{2^{n}-1}$. We also showed that a six 
round KASUMI type permutation is super-pseudorandom (Theorem 5.1). We proved that the advantage is at most $\frac{9 q(q-1)}{2^{n}-1}$.

It is an important open question to prove (or disprove) the super-pseudorandomness of the five round KASUMI type permutation. We conjecture that it is super-pseudorandom.

\section{References}

[1] http://www.3gpp.org/.

[2] 3GPP TS 35.202 v 3.1.1. Specification of the 3GPP confidentiality and integrity algorithms, Document 2: KASUMI specification. Available at http://www. 3gpp.org/tb/other/algorithms.htm.

[3] Evaluation report (version 2.0). Specification of the 3GPP confidentiality and integrity algorithms, Report on the evaluation of 3GPP confidentiality and integrity algorithms. Available at http://www.3gpp.org/tb/other/algorithms.htm.

[4] M. Blunden and A. Escott. Related key attacks on reduced round KASUMI. Fast Software Encryption, FSE 2001, LNCS 2355, pp. 277-285, Springer-Verlag, 2002.

[5] T. Iwata, T. Yagi, and K. Kurosawa. On the pseudorandomness of KASUMI type permutations. The Eighth Australasian Conference on Information Security and Privacy, ACISP 2003, LNCS, 2727, pp. 130-141, Springer-Verlag, 2003.

[6] J. S. Kang, S. U. Shin, D. Hong, and O. Yi. Provable security of KASUMI and 3GPP encryption mode f8. Advances in Cryptology - ASIACRYPT 2001, LNCS 2248, pp. 255-271, Springer-Verlag, 2001.

[7] J. S. Kang, O. Yi, D. Hong, and H. Cho. Pseudorandomness of MISTY-type transformations and the block cipher KASUMI. Information Security and Privacy, The 6th Australasian Conference, ACISP 2001, LNCS 2119, pp. 60-73, Springer-Verlag, 2001.

[8] M. Luby and C. Rackoff. How to construct pseudorandom permutations from pseudorandom functions. SIAM J. Comput., vol. 17, no. 2, pp. 373-386, April 1988.

[9] M. Matsui. New structure of block ciphers with provable security against differential and linear cryptanalysis. Fast Software Encryption, FSE '96, LNCS 1039, pp. 206-218, Springer-Verlag.

[10] M. Matsui. New block encryption algorithm MISTY. Fast Software Encryption, FSE '97, LNCS 1267, pp. 54-68, Springer-Verlag.

[11] K. Sakurai and Y. Zheng. On non-pseudorandomness from block ciphers with provable immunity against linear cryptanalysis. IEICE Trans. Fundamentals, vol. E80-A, no. 1, pp. 19-24, April 1997. 


\section{A Flaws in the proof of $[6]$}

Kang et al. claimed that:

- the four round MISTY type permutation is pseudorandom for adaptive adversaries [6, Theorem 1] and

- the four round KASUMI type permutation is pseudorandom for adaptive adversaries $[6$, Theorem 3].

In this section, we show that both proofs are wrong. In what follows, we use the same notation as in [6].

\section{A.1 Flaws on Theorem 1}

On advantage. In [6, Proof of Theorem 1, p.262], it is stated that

$$
\left|\operatorname{Pr}\left(T_{\Lambda_{n+m}}=\sigma \mid \sigma \notin \operatorname{BAD}\left(f_{1}, f_{2}\right)\right)-\operatorname{Pr}\left(T_{\mathcal{P}_{n+m}}=\sigma\right)\right| \leq \varepsilon_{n, m, q},
$$

and then

$$
\begin{aligned}
\sum_{\sigma \in \Theta} \operatorname{Pr}\left(\sigma \notin \operatorname{BAD}\left(f_{1}, f_{2}\right)\right) \\
\quad \cdot\left|\operatorname{Pr}\left(T_{\Lambda_{n+m}}=\sigma \mid \sigma \notin \operatorname{BAD}\left(f_{1}, f_{2}\right)\right)-\operatorname{Pr}\left(T_{\mathcal{P}_{n+m}}=\sigma\right)\right| \leq \varepsilon_{n, m, q},
\end{aligned}
$$

where $\varepsilon_{n, m, q}=\left\{2^{n+m}\left(2^{n}-1\right)\left(2^{m}-1\right) \cdots\left(2^{n}-q+1\right)\left(2^{m}-q+1\right)\right\}^{-1}$.

However, we can only say that there are at most $1 / \varepsilon_{n, m, q} \sigma$ such that $\sigma \in \Theta$. This implies only that

$$
\begin{aligned}
\sum_{\sigma \in \Theta} \operatorname{Pr}\left(\sigma \notin \operatorname{BAD}\left(f_{1}, f_{2}\right)\right) & \\
\cdot & \left|\operatorname{Pr}\left(T_{\Lambda_{n+m}}=\sigma \mid \sigma \notin \operatorname{BAD}\left(f_{1}, f_{2}\right)\right)-\operatorname{Pr}\left(T_{\mathcal{P}_{n+m}}=\sigma\right)\right| \leq 1
\end{aligned}
$$

and $A D V_{\mathcal{D}}<1$. Hence it does not prove that $A D V_{\mathcal{D}}$ is negligible.

On collision. In $[6$, Lemma $4, \mathrm{p} .261]$, it is stated that

$$
\operatorname{Pr}\left(f_{3}\left(L_{2}^{(i)}\right)=y_{L}^{(i)} \oplus \overline{R_{2}^{(i)}} \text { for } 1 \leq \forall i \leq q\right)=\frac{\left(2^{n}-q\right) !}{\left(2^{n}\right) !}
$$

where:

- $f_{3}$ is a random permutation over $\{0,1\}^{n}$,

- $L_{2}^{(i)}$ is a fixed $n$-bit string such that $L_{2}^{(i)} \neq L_{2}^{(j)}$ for $1 \leq \forall i<\forall j \leq q$,

- $y_{L}^{(i)}$ is a fixed $n$-bit string such that $y_{L}^{(i)} \neq y_{L}^{(j)}$ for $1 \leq \forall i<\forall j \leq q$, and

- $\overline{R_{2}^{(i)}}$ is a fixed $n$-bit string such that $\overline{R_{2}^{(i)}} \neq \overline{R_{2}^{(j)}}$ for $1 \leq \forall i<\forall j \leq q$.

However eq. (26) does not hold because in general, $y_{L}^{(i)} \oplus \overline{R_{2}^{(i)}} \neq y_{L}^{(j)} \oplus \overline{R_{2}^{(j)}}$ does not hold even if $y_{L}^{(i)} \neq y_{L}^{(j)}$ and $\overline{R_{2}^{(i)}} \neq \overline{R_{2}^{(j)}}$. For example, $y_{L}^{(i)}=0^{n}, y_{L}^{(j)}=10^{n-1}, \overline{R_{2}^{(i)}}=0^{n}, \overline{R_{2}^{(j)}}=10^{n-1}$.

Exactly the same problem occurs in the analysis of $f_{4}$ in [6, Lemma 4, p.261]. 


\section{A.2 Flaws on Theorem 3}

In $[6$, p.266] it is stated that "Theorem 3 is proved straightforwardly by the similar process in the proof of Theorem 1." However, the proof of Theorem 1 is wrong as shown above. Therefore, the proof of Theorem 3 is also wrong. (In addition, the proof of Lemma 6 is wrong similarly to above.) 